\title{
Visual structures for achromatic color perceptions
}

\author{
HOWARD R. FLOCK and STEVEN NUSINOWITZ \\ York University, Downsview, Ontario, Canada
}

\begin{abstract}
Accuracy of achromatic perceptions (correctly seeing blacks, grays, and whites) was investigated for three-dimensional rooms, painted matte black or white, presented empty or containing matte or specular, and black, middle gray, or white objects. The rooms were illuminated by a point source at various locations or by extended sources. Observers judged the achromatic color of four walls in the room, and in one experiment the objects in the room, with wide-angle binocular viewing, or judged the far wall under reduction conditions. At issue were questions about absolute and relative achromatic (lightness) constancy, specification of the proximal stimulus for achromatic perceptions, and the relevance of Gilchrist's, Land's, Wallach's, and Helson's theoretical concepts. Despite the three-dimensional spatial arrangements and many variations in experimental conditions, achromatic perceptions were tied to ratios of luminances (contrast ratios or CRs) among surfaces. Moreover, the larger the CR's magnitude, the greater the apparent achromatic difference between surfaces. These achromatic distributions for the perceived colors of the walls were approximately centered on middle gray, when intrasurface luminances were heterogeneous, and on white, when intrasurface luminances were very homogeneous. Despite wide-angle binocular viewing, absolute achromatic constancy occurred only if the CRs were caused by reflectance differences between matte surfaces or within a specular surface. (The stimulus for apparent white could sometimes be an exception to this.) Constancy failed when CRs were caused by illumination gradients on surfaces, even though the scene was three-dimensional and complexly structured. Achromatic constancy depended, therefore, on CRs and on the probability of occurrence of specific physical events. A theory of achromatic perception is described, based on CRs, Helsonian averages, a simple proposition about the illumination, and a concept of nonspatial achromatic segregation.
\end{abstract}

Under normal conditions of viewing real-world scenes, human beings seem to expect that blacks, grays, and whites (called achromatic colors) will correctly appear black, gray, and white. The confidence of human perceivers is such that they seem to expect that even the particular shade of a color will be seen correctly, that, for example, a poor black will be seen as poor black. When the apparent color deviates too dramatically from what it actually is, perceivers seem intrigued, marveling that they can be so much in error. Recently, we had a vivid example of this. Our department's apparatus technician observed through a reduction screen a black surface that he had painted.

When the experiments reported in this paper were conceived and run, we had not anticipated the extent to which Helsonian concepts would describe the results. The patterning of the data in Figures 2 to 4 in this paper is a tribute to Harry Helson and his theoretical legacy. In addition we wish to thank the following students for their assistance in running the experiments: Andrea Birkenfeld, Penny Gershon, Cathy Hill, Kathy Kobata, Roswitha Roese, Carie Ka-Lai Suen, and Chui-Chui Wong. The research was supported in part by grants from the Natural Sciences and Engineering Research Council of Canada and from York University. Much of the data was reported at conferences in 1982 and 1983 (see Flock, 1982, 1983). Requests for reprints should be sent to Howard R. Flock, Department of Psychology, York University, Downsview, Ontario, Canada M3J 1 P3.
He was astonished that it appeared white, not black.

Dramatic errors such as blacks appearing white are often associated with unusual conditions of viewing. Some examples of these conditions are impoverished stimulation, as in using reduction screens (Katz, 1935), deceptive arrangements of light (Gelb, 1929) or deceptive shadow casters (Kardos, 1934), minimal structure in the light, as in the case of simple contrast ratios (Hering, 1905-1911/1964; Wallach, 1948), uncertain and variable spatial organizations (Gilchrist, 1977, 1980). Such errors are generally not expected to occur under normal conditions of complex-scenes viewing. Nevertheless, even under normal conditions of viewing, such extreme errors and less dramatic ones, like blacks appearing gray and grays appearing black, etc., are to be expected, even required, by the theoretical concepts described in this paper. ["Normal viewing" in this paper means two eyes, large field of view, three-dimensional space, and "normal" (viz, not tricky, not deceptive) illumination of that space.]

The topic that we are addressing is that of achromatic color perception, or lightness perception. The topic is frequently dealt with from the point of view of achromatic (lightness) constancy, a discipline concerned with the accuracy of achromatic perception. (We prefer the term "achromatic constancy" to the 
exactly equivalent term "lightness constancy.") Two types of achromatic constancy will be distinguished: one, absolute; the second, relative. Absolute achromatic constancy occurs when achromatic colors appear to be exactly what they are: an actual black that appears black. Relative achromatic constancy occurs when several surfaces that are the same achromatic color appear to be the same color, even if the reported color is in error. For example, several black surfaces may appear the same color, but the seen color may be middle gray instead of black. Relative achromatic constancy also occurs when surfaces that achromatically differ are seen to differ, even if the perceptions are again in error. For example, a middle gray and light gray may be seen, respectively, as light gray and very light gray, a shift upward in the achromatic scale.

Ironically, claims for achromatic constancy are more a matter of belief, faith, and intuition than of controlled empirical demonstration. Based on everyday experience, we "know" that it occurs. The assumption seems to be that if viewing conditions and scenes are normal, achromatic constancy (viz, accurate achromatic perceptions) will prevail to a very high degree. Inherent in that conviction is the intuition that achromatic constancy depends on a great deal of structure and complexity in the visual stimulus. The intuition may or may not be correct. Moreover, even if the intuition is correct, it fails to specify what kind of complexity and structure is required. In this paper, the overall empirical goal is to test the effects on achromatic constancy of four different kinds of stimulus complexity, using simulations of real scenes. Each class of visual stimulus will be distinguished by the physical conditions that produced it. These conditions-illumination gradients, surface reflectances, surface specularity, and a "null structure" - will be shown to produce different expectations and different results, as they pertain to achromatic constancy.

\section{Illumination Gradients}

In the experiments to be described later, the basic experimental situation employed a small room, about $4 \mathrm{ft}$ high, $3 \mathrm{ft}$ wide, and $66 \mathrm{in}$. deep, painted a poor, matte black. That room will also be a topic of consideration in this introduction.

Consider such a room, empty and illuminated by a point source in one corner of the room. The light source would cast complexly different gradients on each wall surface. From any viewing point for the room, the light stimulus would be complexly structured. The structure of the light could be increasingly complicated by filling the room with objects, all painted the same color as the room. Elaborate cast and attached shadows would then be added to the structure of the stimulus, enriching it beyond our intellectual capacity to analyze it.

Would all of this structure and complexity be sufficient to produce a high level of achromatic constancy?
Answering the same question about illumination gradients in a black matte room was a major focus of the experiments we ran. In answer to that same question, Gilchrist (1979; Gilchrist \& Jacobsen, 1984), using a comparable, but smaller, black matte room, said "yes." He supported his answer with empirical data and provided an elaborate set of theoretical speculations to explain the result.

In Gilchrist's experiments, the black matte room, full of black matte objects, was binocularly observed, using a wide-angle view. Overhead, in the left corner, a 200-W bulb differentially illuminated the various surfaces. (A solitary bulb, used as a light source, will hereafter be called a point source. Locating a point source asymmetrically in a space produces different median luminances on the various wall surfaces.) Despite the luminance differences from wall to wall, Gilchrist expected his subjects to see all walls as the same color (perfect relative achromatic constancy), and he expected that color to be black (absolute achromatic constancy). Twenty of 21 subjects in two experiments saw all of the surfaces in the matte black room as having the same achromatic color but "surprised" Gilchrist by reporting that color to be dark gray in the earlier experiment (Gilchrist, 1979) and middle gray in the later experiment (Gilchrist \& Jacobsen, 1984), instead of black.

For achromatic constancy to prevail under these conditions, the visual system would necessarily have to separate the illumination gradients from the underlying reflectance luminances. Gilchrist (1979; Gilchrist, Delman, \& Jacobsen, 1983; Gilchrist \& Jacobsen, (1984) believes that the visual system does just that. A hypothesized visual mechanism, in effect, reads through the illumination gradients to whatever differences or similarities there might be in surface reflectances. Surfaces with the same reflectance would then appear achromatically the same, and surfaces with different reflectances would appear achromatically different. Moreover, for filled spaces, according to Gilchrist, the level of stimulus complexity would require surfaces to appear correctly in their actual colors. (The reflectance of a surface is a term specifying the proportion of the illumination reflected nonspecularly by a surface. In some accounts, perfect white and black surfaces have reflectances of 1.0 and 0 , respectively, with the rest of the achromatic domain lying in between. As a result, the term is often used to indicate the actual achromatic color of a surface, as well as the proportion of incident light that the surface will reflect.)

However interesting and persuasive Gilchrist's theoretical speculations might seem, they ought to rise or fall on the presence or absence of empirical support. As we shall show later, in describing the results of attempted replications, the empirical support may be lacking.

The question on which this discussion has focused concerns a particular kind of visual stimulus. It is composed of luminance differences that are caused 
only by the illumination, not by differences in surface reflectances. In the experiments reported below, these luminance differences (in a black matte room) were varied, using extended (diffuse) sources over the ceiling or point sources at different locations in the room. Diffuse illumination minimized luminance differences among walls; a point source maximized the differences. The complexity of illumination gradients was also varied by filling the empty space with objects that were painted the same matte black as the rest of the room. This variation, however, had no measurable effects on the median luminance values for the wall surfaces.

\section{Measuring a Median Luminance}

Only one aspect of illumination gradients will be investigated in this paper-the median luminance of the gradient on a surface. A wall surface may have exquisite patterns of light distributed over it, caused by multiple sources of light. Only the median of that light show will concern us. This narrow interpretation of illumination gradients is intended in any mention or discussion of them. Correspondingly, the method used to represent illumination gradients on a surface was equally restricted. Forty to 50 readings were taken for the visible regions of each wall surface, using a Spectra Pritchard photometer with a 6-min probe. The median of those readings became that surface's luminance. There were four visible "walls": the two side walls of the room, the far wall, and the floor. When a set of objects was placed in the room, the set was collectively treated as a single surface. The median luminance of that "object surface" became a fifth surface. (There were six sets of wood objects: three matte and three specular, painted black, white, and middle gray. The median luminance measures of these objects were determined in part by any highlights or attached shadows on the surfaces.)

The concept of luminance ratios will be introduced below. A ratio between two luminances (contrast ratio or CR) is formed, in our meaning of the term, only if there is an abrupt change in the light, as at the intersection between two surfaces or as in a comparison between two surfaces with different median luminances. A luminance ratio, in this sense, is not formed on a surface from the gradual, or often dramatic, change in the light that is typical of illumination gradients.

\section{Predictive Effects of Illumination Gradients on Relative Achromatic Constancy}

The different experimental arrangements used in our experiments to produce and vary illumination gradients have already been mentioned. The effect of these experimental variations on achromatic perceptions could not be directly predicted from the empirical literature on lightness (achromatic) constancy.
Except for Gilchrist's expectations that all of the black surfaces should appear black under at least some of the conditions that we used, the empirical literature was not explicitly helpful. Theories of lightness (achromatic) constancy and their explanatory mechanisms had been tested in situations that, at best, were limited abstractions from real-world scenes. Nevertheless, explanatory mechanisms from three achromatic theories seemed particularly relevant and generalizable to this discussion. Their defining data, however, tended to be based on flat, matte, untextured surfaces, not textured surfaces arranged threedimensionally, as in the experiments to be described below.

The three theories were Land's (1977; Land \& McCann, 1971) concept of edge ratios, Wallach's $(1948,1963)$ concept of contrast ratios (CRs), and Helson's $(1938,1947,1964)$ concept of adaptation level (AL). Although conceptually different, each of these theories can be shown to make approximately the same predictions about relative achromatic constancy, given illumination gradients in the black matte room. In addition, Helson's theory is reasonably explicit in predicting whether absolute achromatic constancy will occur.

Land (1977; Land \& McCann, 1971) has implied that slowly changing gradients of light across a surface, as in the case of many illumination gradients, have no perceptual effects. Despite that, Land would expect that such illumination gradients could have pronounced effects at edges between surfaces. This effect of illumination gradients at edges can be observed in any space that is illuminated by a point source. At an intersection of two surfaces, the one oriented away from the source will have a lower luminance than the one facing the source. Provided that the luminance difference across edges has at least a magnitude of 1.003 to 1.0 , then it will have achromatic consequences in Land's (1977) theory. The difference, expressed as a ratio of luminances, would cause the surface with higher luminance to be seen as achromatically lighter. The integration of all these ratios for all surface intersections in a scene would specify a distribution of luminance ratios and a corresponding distribution of apparent achromatic color. Under these circumstances, with no reflectance differences but with luminance differences at edges caused solely by the illumination, Land would have to predict, we believe, corresponding differences in apparent surface color and therefore no achromatic constancy.

Land's edge ratios belong to a family of analytic concepts called contrast ratios (CRs). The CR has a venerable history, dating back to Hess and Pretori (1896/1970), Hering (1905-1911/1964), and others; and it has had its share of controversy. (For an account of some of the controversy, see Flock, 1974.) Wallach 
$(1948,1963)$ is frequently associated with the current theoretical status of $\mathrm{CR}$. He has proposed $\mathrm{CR}$ as the explanatory mechanism for lightness constancy. Whenever one region of luminance (the target area) differs from a second region of luminance (background, surround, or nearby area) that is spatially adjacent, the ratio of the two luminances forms a $\mathrm{CR}$. The magnitude of the ratio determines the relative difference in the apparent achromatic colors.

When luminance arrays are complicated, as in the black matte room with its complex illumination gradients, it is not clear how Wallach might quantify background luminance so as to produce a CR for each surface. This problem had to be solved in order to place our own data in an appropriate conceptual framework. The solution we adopted depended primarily on Helson's (1964) concept of an average luminance or AL (adaptation level). (For an analysis of some of the problems in specifying AL for a complex scene such as the black matte room and for a description of some of the strengths and weaknesses of Helsonian theory as it pertains to achromatic perception, see Flock, 1972. The same problems described there for specifying AL would also apply in trying to extend Wallach's concept of background luminance to a complex scene.)

The application of Helsonian theory to the black matte room first required the calculation of an "average" of all luminances in the scene. The arithmetic average of the four median surface-luminances became the $\mathrm{AL}$ or overall representative luminance. This procedure produced a representative overall luminance for the scene, to be referred to hereafter by the acronym ROLS. The ROLS approximated Helson's concept of AL and departed only slightly from Wallach's concept of a background (or surround) luminance. (Presumably, Wallach would have excluded from each average the luminance of the target region. For most real-world scenes, that would produce only a trivial difference between the two concepts.)

Finally, CR for each surface in a scene was the ratio of a surface's luminance to ROLS. The values of these Helsonian-type CRs would be similar to the values of Land's edge ratios and Wallach's CRs. For all three theories, apparent achromatic differences, caused by the illumination, would be expected to correlate to the magnitude of the CRs. Plotting the achromatic appearance of surfaces against their CRs (or their edge ratios) should produce a smooth, rising function through the data points. This means that Helson, Land, and Wallach would predict no relative achromatic constancy if the illumination produced discriminable differences in surface luminances.

\section{Predictive Effects of Illumination Gradients on Absolute Achromatic Constancy}

For the black matte room, the achromatic theory associated with Wallach does not explicitly predict what specific color will be seen. Land (1977, pp. 120 122) implies that the region of highest luminance in a scene will be seen as white. This could mean that at least one of the walls in the black matte room, regardless of how it is illuminated, should appear white. Gilchrist expected the surfaces to appear black, but that prediction may have been gratuitous, since it was not clear how it was derived from or predicted by his theory. In comparison, Helsonian theory makes unequivocal predictions.

Under diffuse illumination, with light sources spread over the ceiling, the black matte walls would have more or less the same luminance. As a result, surface luminances would deviate only narrowly from the luminance value of the ROLS. According to Helsonian theory, any surface that has a luminance approximately equal to the ROLS will, in general, appear middle gray. Under diffuse illumination, therefore, the walls of the black matte room should appear middle gray, an instance of perfect relative achromatic constancy, but a failure of absolute achromatic constancy.

With a point source, the median surface luminances would differ, depending on the location of the source. Any surface luminance close in value to the ROLS would, according to Helsonian theory, appear middle gray. As surface luminances deviated from the ROLS, achromatic color would correspondingly deviate from middle gray. With sufficient differences in luminance, walls that were actually the same color might appear to vary from white to black. Thus, neither relative nor absolute achromatic constancy would be expected to occur. (Using a 200-W bulb, Gilchrist and Jacobsen, 1984, reported that all surfaces appeared the same middle gray, thereby contradicting Helsonian expectations. However, their "room" was relatively small, being $2 \mathrm{ft}$ on a side, which might have diffused the light; the walls of their room tended to be occluded from view by a clutter of objects; and their psychophysical method may have led the subjects to ignore the wall surfaces in favor of the many objects they were asked to judge.)

\section{Reflectance Differences}

In this discussion, the structure and complexity of the visual stimulus have been determined solely by the illumination. For such a stimulus, relative achromatic constancy can occur, but probably not absolute achromatic constancy. For absolute achromatic constancy to occur, a second kind of visual stimulus is generally required. Differences in the reflectance of surfaces must be represented in the structure of the visual stimulus. To accomplish this, white matte objects were placed in the black matte room. Although wall luminances were unaltered by such arrangements, the white "object surface" expanded the range of median luminances in the space by factors greater than 35 to 1 . With that expanded range of median luminances and the corresponding increase in mag- 
nitude of the CRs, the black walls that in Gilchrist's experiment had appeared middle gray might now be expected to appear black. That would be perfect absolute achromatic constancy.

\section{Specularity of Surfaces}

Both illumination gradients and differences in the reflectance of matte surfaces can produce large CRs and corresponding effects on achromatic responses. Whether achromatic constancy will occur depends less on the visual system and more on the kind of physical events that produced the CRs. This would make achromatic constancy an adventitious result of chance physical events. Nevertheless, the probability of achromatic constancy in the real world is probably very high, because of two facts about realworld ecological events. First, spaces with the same reflectance for each surface are rare events in the real world. More typically, surfaces have a range of reflectances. Second, and equally important, the real world is not composed of the matte surfaces that are often endemic to perceptual research. Virtually every real-world object is, as Hunter (1975) has pointed out, more or less specular (glossy) as well as more or less matte. Specular objects, as we shall explain, carry their own CRs as a distinguishing feature of their specularity.

A specular surface that is opaque, nonmetalic, and achromatic has two different reflectances. Its diffuse reflectance is approximately like the reflectance of a matte surface. That is to say, its diffuse reflectance approximately specifies the proportion of the incident light that will be reflected. The achromatic color of the specular surface (and its texture) is associated with (and coded by) the diffuse reflectance, just as in the case of matte surfaces.

The specular surface has a second, dramatically different reflectance, called the specular reflectance. Because of these specular reflectances, specular surfaces appear to have gloss, shine, luster, sheen, sparkle, and highlights (Beck, 1981; Hunter, 1975). The specular reflection is, in fact, a mirror image of (primary and secondary) light sources that are external to the surface. These images may vary from being near perfect, like those in "real" mirrors, to being perfectly degraded, as found in what are called matte surfaces (Flock, 1984). The specular surface presents to the eye its diffuse reflection superimposed on a "picture" of the total surrounds. The specular surface is potentially a microcosm, encapsulating not only a total view of the space in which it lies, but also the variables that constitute the achromatic theory presented in this introduction. As a result, the specular surface may contain that range of reflectances which in the earlier discussion of matte surfaces was associated with absolute achromatic constancy.

The CRs between the specular and diffuse reflections are an inherent, built-in feature of the specular surface. This feature is theoretically associated with the specular object's apparent achromatic color. In explanation, the magnitudes of the specular CRs tend to be constant as illumination is increased. The magnitudes decrease, however, as the achromatic color of the specular surface is altered from black to white. As a result, the magnitudes of the specular CRs would theoretically be correlated with both the actual and the apparent achromatic color of the surface. Black objects that are moderately specular, for example, produce, on average, high-intensity specular reflections and low-intensity diffuse reflections. For white objects, the luminances of the two reflections are more similar. In this sense, the CRs inherent to specular objects would be correlated to the object's color and could theoretically specify the actual color of the object.

The prediction is unambiguous and easily tested. In one experiment, four sets of objects, separately displayed on the floor of the black matte room, were presented to four separate groups of subjects. The four sets of objects were specular and matte, middle gray and white. They were illuminated by point and extended sources. The specular objects mirrored the illuminating source as well as the black walls with their illumination gradients. Unlike those in all the other experiments in this paper, the subjects judged the achromatic color of just the objects, not the walls. Both the white matte and specular objects, contrasted to the black walls, should appear white. A high level of achromatic constancy might also be expected of the middle gray specular objects, with their built-in CRs, but would not be expected of the middle-gray matte objects. (Beck, 1964, observed this result in a study that has been virtually unique in the perceptual literature for its investigation of the effect of specularity on apparent achromatic color.)

We were concerned with some other more problematic questions about specularity. Very black specular objects were placed on the floor of the black matte room. The high-intensity specular highlights, mirroring the light sources, and the low-intensity diffuse reflectance of the black specular objects would greatly expand the luminance ranges in the space. The luminances of the black walls would lie somewhere within that range. Of particular interest was the possibility that the intense highlights would form CRs with the wall surfaces of the room. If that were the case, then the walls would be expected to appear black. In other words, intense highlights might function like white matte objects placed in the room.

Even if the intense specular highlights did not interact generally with the vertical walls of the room, the literature on simultaneous brightness-contrast would predict an interaction with the floor on which the objects sat (Leibowitz, Mote, \& Thurlow, 1953). The objects were radially adjacent to, and surrounded by, an angularly large contrasting field (i.e., the floor). 
For these conditions, the intense highlights might be expected to darken the floor more than they darkened the vertical walls. Correspondingly, the large angular expanse of white matte objects placed on the floor, in the experiment mentioned earlier, should also produce differential darkening of the floor, as compared with the appearance of the vertical walls. Such conclusions would be supported by contrast theories that do not require strict coplanarity of surfaces. In those theories, however, contrast effects would be expected to diminish as surfaces were increasingly separated, laterally or radially (Beck, 1965; Koffka, 1935; Mershon, 1972). Other theories require the apparent coplanarity of surfaces for the formation of CRs and production of interactive effects (Gilchrist, 1980).

Actually, all of the experiments reported in this paper might be construed to be tests of coplanar theories of contrast. The achromatic theory presented in this paper could not, however, easily accommodate vigorous empirical findings that demonstrated the necessity of invoking coplanarity to explain contrast effects.

\section{CR: A Determining Stimulus}

The three classes of visual stimuli that have been described have been distinguished by the conditions that produced them: illumination gradients and the reflectances of matte and specular surfaces. Despite the differences in these conditions, the three different stimulus structures embed commonplace ratios of luminances. The CRs that have been described are indistinguishable with regard to how they are produced or to the particular visual structure in which they are embedded. The theoretical claim, generalized from the three theories that have been reviewed (viz, by Land, Wallach, and Helson), is that CRs, rather than the complex visual structures in which they are embedded, determine achromatic appearances.

The CR is proposed, therefore, as a prime visual stimulus for achromatic perceptions. Also, whether achromatic constancy occurs or not depends on what kind of physical events are in correspondence with the prevailing CRs. Absolute achromatic constancy depends, we have argued, on reflectance differences among matte surfaces or within specular surfaces. Relative achromatic constancy depends only on diffuse illumination of surfaces, irrespective of their reflectances. Complexity and structure of the visual stimulus do not in themselves determine whether achromatic constancy will occur.

In the experiments described below, the effects on achromatic constancy of the three different classes of visual stimulus were examined. At the same time, these visual structures were looked at from the point of view of their embedded CRs. The theoretical expectation was that achromatic appearances are determined by CRs. Neither the complexity of the conditions nor the different visual structures that pro- duced the CRs were expected to determine achromatic perceptions. The theoretical predictions, therefore, were unambiguous.

In all but one of the experiments with the black matte room, subjects judged the achromatic color of four walls of a black matte room. Illumination gradients were varied by changing the location of a point source and by using extended sources. The visual stimuli were complicated by placing various matte and specular objects in the room. In one experiment, in order to evaluate the effect of specularity on the apparent color of specular objects, subjects judged just the color of middle-gray and white specular and matte objects.

\section{A Null Structure}

In this discussion, CRs have been indicated as necessary and sufficient conditions for achromatic appearances. In addition, for absolute achromatic constancy to occur, CRs formed by reflectance differences between matte surfaces or within specular surfaces are required, as we have claimed. Nevertheless, there is at least one instance in which absolute achromatic constancy may occur even though CRs and reflectance differences seemingly do not occur. Gilchrist and Jacobsen (1984) described a white matte room as appearing white, even though no obvious CR was present. That experiment was repeated and is described below. Wall luminances were very homogeneous. The subjects judged the achromatic color of the walls of the white matte room under full binocular viewing. In addition to this, the subjects judged, in other experiments, the achromatic color of the far walls in the black and white rooms, under reduction conditions.

The stimulus "structure" in both of these sets of experiments will be discussed below. For the present it should be noted that they have in common an ambiguity as to the background out of which a CR is formed. If there is no relevant background, there is no $C R$. If there is no $C R$, the stimulus structure is "null." As a result, the stimulus situation for the white matte room and for the reduction conditions is different from situations in which a CR is easily hypothesized.

Finally, there was a methodological experiment that was useful to the analysis of the data. That is reported in the Procedure section.

\section{METHOD}

\section{Apparatus}

Two rooms, with the dimensions shown in Figure 1, were constructed of a smooth grade of fir plywood. The interior wall surfaces of one room were painted matte black, equivalent to a Munsell 2.3. The second room was painted matte white, equivalent to a Munsell 9.3. (The paint was Glidden interior-exterior enamel flatblack 908 and flat-white 5700 .)

A hooded aperture was centered on one wall, to give subjects a binocular view of the other four walls (left wall, right wall, far 


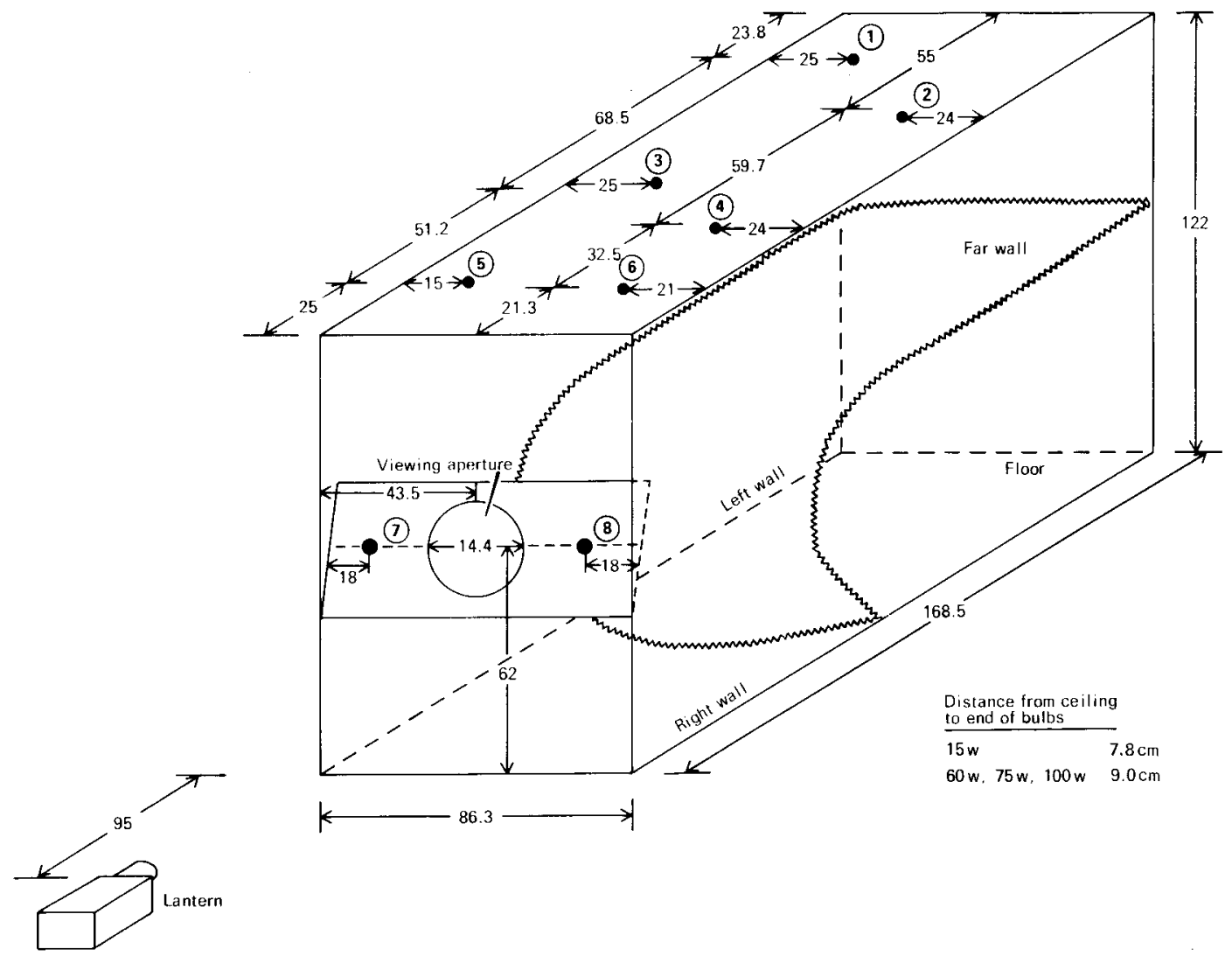

Figure 1. The experimental chamber. The wavy line indicates roughly the visible areas of four wall surfaces. The circled numbers indicate socket-locations for bulbs; all other numbers are measures in cen timeters.

wall, and floor) but not of the ceiling. The view of the room, 60 deg laterally and 45 deg vertically, included more than half of the far wall and roughly three-fifths of each of the two side walls and floor. Sketched into Figure 1, with a wavy line, is an approximate, scaled depiction of the visible wall surfaces. By placing a reduction screen in front of the viewing hood and then a monocular aperture $25 \mathrm{~cm}$ from that screen, the subjects were also provided a 10-deg view of the far wall of each room under reduction conditions (with chin- and headrests to control head position).

Five different lighting arrangements were used to illuminate the black matte space. These are described in Table 1. Arrangement I, for example, used a 75-W point source, located over the far wall in the left corner. (The location is determined by noting the socketlocation in Table 1 and then looking it up in Figure 1.) In Arrangement III, a 100-W bulb was located overhead in the left corner. An example of extended sources (i.e., diffuse illumination) is Arrangement IV. Seven of the eight socket positions in Figure 1, including socket locations 7 and 8 on the back side of the viewing hood, were occupied by $15-\mathrm{W}$ bulbs. When, as in this case, bulbs occupied seven or eight socket positions, the total light source will be referred to as an extended or diffuse source, and the resulting illumination as diffuse illumination.

One exceptional illumination condition was Arrangement II, for which a lantern was used, located $95 \mathrm{~cm}$ beyond the black matte room. It is shown in Figure 1. The center of its directed beam was $1 \mathrm{~cm}$ above floor level and $3 \mathrm{~cm}$ in from the left wall. This source was fully visible to the subjects, although it was behind the subjects, on the left, while they were making observations. When this source was used, the black velvet curtain below the viewing hood was opened. Under all other experimental conditions, illumination sources were not visible to the subjects at any time.

Arrangement VI applied to the white matte room. The 5-W bulb was located on the back side of the viewing hood. Its output and the reflecting power of the white walls were sufficient to produce relatively high, relatively homogeneous luminances of the four white wall surfaces.

These lighting arrangements produced varied luminances over the visible areas of the four black matte walls. The medians of those "visible" luminances are given in Table 1. Also given there is the luminance of the far wall under reduction conditions and the median luminances of the objects that were used in three experiments.

Two sets of wood objects were painted either matte black (Munsell 2.3) or matte white (Munsell 9.3), using the same paint applied to the wall surfaces. A third matte set was painted middle gray (Munsell 5.5). The wood objects were: two salad bowls, with diameters and heights of 27 and $9 \mathrm{~cm}$, respectively; a salad spoon, $20 \mathrm{~cm}$ long; and two round objects about $7 \mathrm{~cm}$ in diameter, shaped like an egg and an apple. When used in the black matte room, a set of these wood objects was spread over the floor in the farther part of the space. They were arranged so as not to block a view of the dihedral intersections of the various wall surfaces or occlude a continuous span of floor from foreground to far wall. The set of objects was considered to be a single "object surface." Median luminances for each set are given in a note below Table 1 .

In addition to these matte objects, three other sets of identical objects were painted specular black (Munsell 0.5), white (Munsell 9.2), and middle gray (Munsell 5.3). (The paint was Glidden interiorexterior enamel, gloss white 900 and gloss black 909). Various 
Table 1

Six Different Illumination Arrangements, Including Point and Extended Sources, for Black and White Matte Rooms, With Median Luminances for Far Wall Under Reduction and All Four Walls and Six Sets of Objects Under Full Binocular View, as Well as Maximum (MAX) and Minimum (MIN) Luminance Values

\begin{tabular}{|c|c|c|c|c|c|c|c|c|}
\hline \multirow[b]{3}{*}{ Type of Illumination (Bulbs)* } & \multirow[b]{3}{*}{$\begin{array}{l}\text { Socket } \\
\text { Location }\end{array}$} & \multicolumn{7}{|c|}{ Luminances in Foot Lamberts } \\
\hline & & \multicolumn{3}{|l|}{ Reduction } & \multicolumn{4}{|c|}{ Binocular Full View } \\
\hline & & $\begin{array}{l}\text { Far } \\
\text { Wall }\end{array}$ & $\begin{array}{l}\text { Far } \\
\text { Wall }\end{array}$ & Floor & $\begin{array}{l}\text { Right } \\
\text { Wall }\end{array}$ & $\begin{array}{l}\text { Left } \\
\text { Wall }\end{array}$ & $\operatorname{Max}$ & Min \\
\hline I. Point over far wall (1 $75-$ W) & 1 & .24 & .19 & .35 & .22 & .20 & .86 & .08 \\
\hline II. Point at floor level (1 200-W) & & & .09 & .004 & .022 & .013 & .14 & .003 \\
\hline III. Point overhead $(1100-W)^{* *}$ & 5 & & .20 & .19 & .17 & .09 & .35 & .04 \\
\hline IV. Extended (7 $15-\mathrm{W})^{* *}$ & $1-4 ; 6-8$ & .17 & .16 & .17 & .14 & .14 & .33 & .06 \\
\hline V. High intensity extended $(2100-W ; 675-W)$ & $1-8$ & 1.55 & 1.45 & 1.60 & 1.08 & 1.02 & 3.05 & .65 \\
\hline VI. White matte room (1 5-W) & 8 & 1.70 & 1.65 & 1.60 & 1.60 & 1.65 & 2.10 & 1.35 \\
\hline
\end{tabular}

*For I, III, IV and V, bulbs were incandescent GE, Shadow-Ban. For VI, clear GE incandescent lamp. For II, a lantern, with a directed beam equivalent to a 200-W bulb. $\quad{ }^{*}$ Under this illumination, the space was empty or filled with black, middle gray, or white, and matte or specular objects. For Arrangements III (point source) and IV (extended source), the median luminances for matte objects were, respectively: black, 0.20 and $0.18 \mathrm{fL}$; middle gray, 1.7 and $1.2 \mathrm{fL} ;$ white, 7.1 and $5.1 \mathrm{fL}$. For specular objects, the corresponding values were: black, 0.042 and $0.067 \mathrm{fL}$; middle gray, 1.5 and $1.2 \mathrm{fL}$; white, 7.5 and $5.8 \mathrm{fL}$. Also, the most intense highlights on the specular objects for the same point and extended sources were: black, 160 and $22 \mathrm{fL} ;$ middle gray, 140 and $23 \mathrm{fL}$; white, 150 and $38 \mathrm{fL}$. For comparison purposes, the maximal luminances on the matte objects for the same point and extended sources were: black, 0.49 and $0.33 \mathrm{fL}$; middle gray, 2.1 and $1.6 \mathrm{fL}$; white, 9.1 and $6.3 \mathrm{fL}$.

luminance measures for these specular objects are also given in a footnote to Table 1 . The values there will not necessarily agree with relative Munsell reflectances. That is because attached shadows, gradients, multiple reflections, highlights, etc., on the surfaces contributed in determining the median luminances. As a result, the median luminances for specular surfaces given in Table 1 should be regarded as rough indicators of the median light, rather than precise measures of it. This warning applies to a lesser extent for all of the measures in Table 1.

\section{Procedure}

The subjects were trained to use 11 different achromatic response categories: very white, white, poor white, very light gray, light gray, middle gray, dark gray, very dark gray, poor black, black, and very black. The training consisted of having the subject examine a set of 32 quarter-step neutral Munsell chips, from 1.75 to 9.5 , under room illumination. Using the 11 response-categories, the subject described various chips that were designated by the experimenter.

In a methodological experiment, an independent group of 14 subjects examined the set of 32 Munsell chips. Their task was to specify which Munsell chip best conformed to each of the 11 response-categories. Their median responses, using extrapolations between Munsell values when necessary, were: very white, 9.5; white, 9.15; poor white, 8.0; boundary between white and gray, 7.2; very light gray, 7.0; light gray, 6.3; middle gray, 5.0; dark gray, 3.15; very dark gray, 2.75; boundary between gray and black, 2.7; poor black, 2.4; black, 1.75; very black, off-scale, estimated to be 1.50 or less. In the analysis and graphing of data, each response was first translated to these Munsell equivalents. (The 11 response-categories and their Munsell equivalents are shown on the ordinate of Figure 2, below.)

During an experimental trial, a subject examined the four black matte walls under full binocular view and judged their achromatic color, using the 11 response categories. If two or more walls appeared the same, the subject was allowed to say so. In four of the experiments, the room was empty or contained black matte, black specular, or white matte objects. In all of these four experiments, the room was illuminated by the same point source on one trial and by the same diffuse source on a second trial, the order being counterbalanced over subjects. Each subject over the two trials made a total of eight judgments. Between each of the two trials there was a 60-sec pause in the darkness of the experimental room.
These four experiments are listed in Table 2 as Experiments 1-4. (The table itself is described just below.)

In four other experiments (Experiments 5-8 in Table 2), the subject first judged the achromatic color of the black matte far wall under reduction conditions (a single judgment). This was followed by a second trial with full binocular view of the room and judgments of the four walls. In all four experiments, the room was empty and was illuminated by either one of two extended sources or either one of two point sources. The subjects made a total of five judgments in the two trials. Other procedures were exactly the same as for Experiments 1-4.

A ninth experiment (Experiment 9 in Table 2) was composed of four separate groups of subjects. For each group, the objects in the room were different-white matte, white specular, middlegray matte, or middle-gray specular. Each of the groups judged the apparent color of the objects. Otherwise, the conditions were precisely the same as for Experiments 1-4.

In Experiment 10, under reduction, the subjects judged both the far wall and the blackness of the darkness of the surrounding reduction screen. Finally, in an 11 th experiment (Experiment 11 in Table 2), an empty white room was employed. It was viewed first under reduction and then with a full binocular view. The general procedures were as in Experiments 5-8.

In Table 2, the codes refer to Figures 2 and 4 and should be ignored at this time. The Roman numerals refer to the illumination conditions in Table 1. As an example, Experiment 4 involved black specular objects in the black matte room, which was illuminated by a $100-\mathrm{W}$ point source overhead in the left corner for one trial and by diffuse illumination with seven 15 -W bulbs for the other trial. Twenty subjects made four achromatic judgments during each of the trials.

A deliberate effort was made to produce cognitive uncertainty. In the instructions, the subjects were told that the walls of the room were mechanically changeable, as in a theater. They were told to expect walls that might be all the same, all different, or some the same and some different in achromatic color. Before each of the two trials of the subject's session, a highly audible, whirring motor was activated, presumptively "'altering surfaces," while the experimenter waited in eloquent silence. (In fact, before each trial the only change was to press a switch to alter illumination or to shift from reduction conditions to full viewing.)

An experimental session, including training, lasted $15 \mathrm{~min}$. The two experimental trials were run in darkness. 
Table 2

Experimental Design for Eleven Experiments

\begin{tabular}{|c|c|c|c|c|c|}
\hline Experiment & Codes & \multicolumn{2}{|c|}{ Type of Illuminating Source } & Type of Object in Room & $\mathrm{N}$ \\
\hline \multicolumn{6}{|c|}{ Black Matte Room } \\
\hline 1 & $\mathbf{A}, \mathbf{H}$ & III. Point* & IV. Diffuse & None & 17 \\
\hline 2 & $\mathrm{~B}, \mathrm{I}$ & III. Point* & IV. Diffuse & Black matte & 20 \\
\hline 3 & $F, K$ & III. Point* & IV. Diffuse & White matte & 22 \\
\hline 4 & $\mathrm{C}, \mathrm{J}$ & III. Point* & IV. Diffuse & Black specular & 20 \\
\hline 5 & $\mathrm{~N}, \mathrm{G}$ & IV. Reduction & IV. Diffuse & None & 18 \\
\hline 6 & $\mathbf{N}, \mathbf{D}$ & I. Reduction & I. Point & None & 12 \\
\hline 7 & $\mathrm{~N}, \mathrm{E}$ & V. Reduction & II. Point & None & 10 \\
\hline 8 & N, L & V. Reduction & V. Diffuse & None & 12 \\
\hline $9 a$ & & III. Point* & IV. Diffuse & White matte & 18 \\
\hline $9 b$ & & III. Point* & IV. Diffuse & White specular & 18 \\
\hline $9 \mathrm{c}$ & & III. Point* & IV. Diffuse & Middle-gray matte & 18 \\
\hline $9 \mathrm{~d}$ & & III. Point* & IV. Diffuse & Middle-gray specular & 18 \\
\hline 10 & & IV. Reduction & & & 10 \\
\hline \multicolumn{6}{|c|}{ White Matte Room } \\
\hline \multirow[t]{2}{*}{11} & $\mathbf{N}, \mathbf{M}$ & VI. Reduction & VI. Diffuse & Empty & 14 \\
\hline & & & & Total N & 227 \\
\hline
\end{tabular}

Note-The letter codes refer to functions in Figure 2. The Roman numeral codes refer to illumination conditions listed in Table 1. *The order of presentation, as between point and diffuse sources, was counterbalanced. All other experiments occurred in the indicated order. For reduction conditions, subjects made a single monocular judgment of the far wall (using head restraints). For each of the illumination conditions, under binocular viewing, the subjects made either four judgments, one each for the four wall surfaces (Experiments 1.8 and 11) or one judgment of the objects in the room (Experiment 9). In Experiment 10, under reduction, the subjects judged only the far wall and the darkness of the surrounding screen.

Experiments 1-8 in Table 2 were run at the same time and therefore will be treated as a single unit of data in the analyses and discussion below. The results for Experiments 9-11, which were collected at a later time, plus those for three other experiments with specular objects, which have not been mentioned in this section, will be introduced separately at appropriate places in the text.

\section{Subjects}

For the 12 experiments (including the methodological experiment) that are described above, 241 students at York University were recruited from three introductory courses in psychology. The median age of the 96 men and 145 women was 20 years. (The disproportionate number of women reflected the imbalance in the number of men and women in the courses.) All subjects were naive about the experiments. Each subject had at least 20/20 corrected vision in both eyes.

\section{RESULTS AND DISCUSSION}

\section{Relative Achromatic Constancy}

A general failure of relative achromatic (lightness) constancy occurred over Experiments 1-8. This failure was particularly striking when point sources, illuminating a space, cast very different illumination gradients on each wall. In general, however, any luminance difference between walls, however produced, generated different achromatic appearances. Some simple statistics illustrate this.

An observer's responses to the achromatic color of the four walls were plotted against the median luminances of the walls. Over all experiments with point sources, there were 101 different subjects and thus 101 of these achromatic functions. Of the 101 functions, $87 \%$ were positively rising functions (with and without flat segments in them). This means that, for $87 \%$ of the observers, two or more of the four surfaces appeared achromatically different. Furthermore, the achromatic differences were in the same direction as the luminance differences. (The remaining $13 \%$ saw all surfaces as having the same color.)

Under diffuse illumination, the luminance differences between walls were small in magnitude. Despite that, 43 of 123 achromatic functions, or $35 \%$, were rising functions, also correlated with the direction of changes in luminance. (For the other 80 functions, all surfaces were seen as having the same color.) Out of the grand total of 224 functions, no overall negative functions occurred. There were, however, 19 reversals in pairs of judgments, scattered among the 224 functions, but more concentrated under conditions of diffuse illumination.

The results are unambiguous. Relative achromatic (lightness) constancy did not occur when, because of the illumination, surfaces with the same reflectance had diffferent luminances.

This finding may seem particularly impressive, given a deliberate procedural bias that was introduced. The observers were allowed to use an "equal" category, to say, in short, that all surfaces appeared to have the same color. The very existence of such a "same" category may have persuaded some observers to use it, even though they noticed differences. The bias, therefore, was in the direction of increasing "same" responses. Our theoretical expectations were in the opposite direction-to find "different" responses. The bias, as a result, seemed acceptable. 
Moreover, the experiments were an attempt to replicate Gilchrist's results, and he used a "same" category.

Despite the availability of the "same" category, the subjects reported achromatic differences when luminance differences occurred. This was in stark contrast to Gilchrist's results. Although 20 of his 21 subjects (combining Gilchrist, 1979, with Gilchrist \& Jacobsen, 1984) used the "same" category under his single point-source condition, only 13 of 101 subjects used it in our experiments, under comparable conditions.

The most dramatic single failure of achromatic constancy in these experiments occurred in the experiment with the point source near floor level, behind and to the left of the subject. Each subject agreed that the far wall appeared poor white, the floor appeared very black, and the sidewalls appeared poor black and dark gray. This condition exemplified our earlier statement that black surfaces can be made to appear both black and white, even under "normal" conditions. This experiment is described by Func- tion $\mathrm{E}$ in Figure 2. (In the figure, median achromatic responses, expressed as Munsell values, are plotted against median luminances, expressed in logs. Each line in the figure describes the results for a different experimental condition. These are explained in the table to the figure and in the discussion below. The letter-codes in the figures are also explained by $\mathrm{Ta}$ ble 2.)

\section{Diffuse versus Point-Source Illumination}

Luminances were more similar, from wall to wall, under diffuse illumination than under point-source illumination. This increased homogeneity of luminances should favor relative achromatic constancy. As a result, achromatic functions should have less rise under diffuse than under point-source illumination. That expectation was satisfied.

The result is clearly evident in Figure 2, where Functions $\mathrm{H}$ to $\mathrm{J}$ for diffuse illumination have less rise than Functions $A$ to $C$ for point sources, and

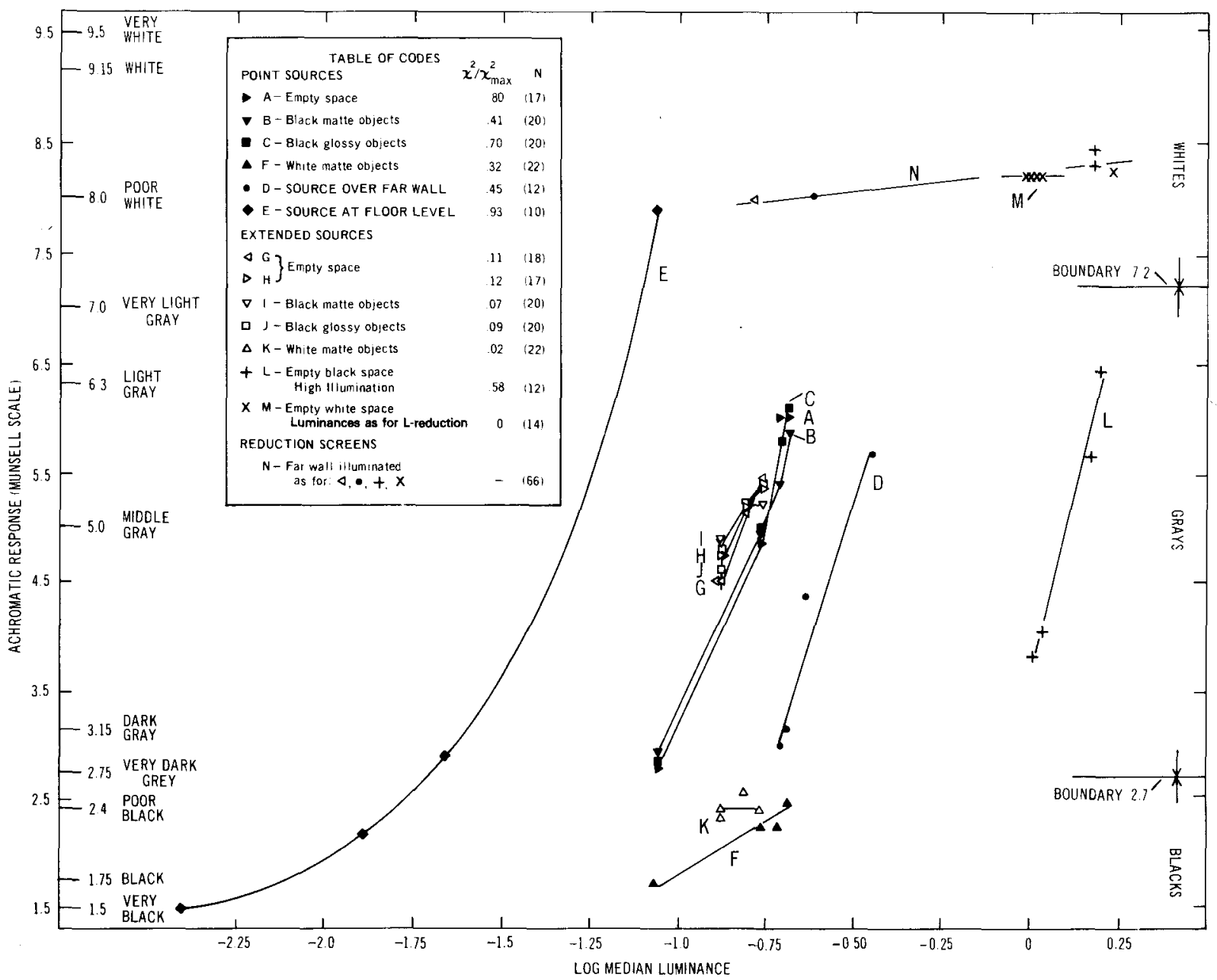

Figure 2. Fourteen achromatic functions for nine experiments, relating median achromatic response to median surface luminance. 
Function $\mathrm{K}$ (diffuse) has less rise than Function $\mathrm{F}$ (point source). To produce these functions, 79 subjects judged the four walls under both diffuse and point-source illumination. For 59 of the subjects, the function had a greater rise (i.e., covered more response categories) for point sources than for diffuse illumination; for a 60 th subject, the result was in the other direction; and there were 19 ties. This result, a 59-1 split, with a chance probability close to $.5^{59}$, is unambiguous in its support of the hypothesis. Diffuse illumination does increase the probability that relative achromatic constancy will occur.

\section{Filled versus Empty Space}

"Filling" the black matte space with black matte objects may be presumed to complicate the light stimulus without altering the median luminances of the wall surfaces or changing the total range of median luminances in the space. As a result, the achromatic appearances of the walls should be the same for both empty and filled space.

The results are shown in Figure 2. Functions A (empty space) and B (filled space) for point-source illumination are virtually congruent; Functions G and $H$ (empty space) and I (filled space) for diffuse illumination are also virtually congruent. (Statistically, within each subset, the smallest probability associated with the Kruskal-Wallis test statistic, $\mathrm{H}$, corrected for ties, was 0.30 .) Thus, the appearance of the black matte walls was unaffected by the presence or absence of black matte objects.

\section{Effect of Black Specular Objects on Surrounding Walls}

In Figure 2, Functions $C$ and $J$ are visibly (and were statistically) congruent with their respective subsets. Functions $\mathrm{C}$ and $\mathrm{J}$ were produced by placing black specular objects in the space. The results were virtually identical to what occurred when the room was empty or filled with black matte objects. Despite the high-intensity specular images on the objects and the large theoretical CRs between those highlights and the black matte walls, the specular highlights seemed to have no darkening effect on the achromatic appearances of the walls. Presumably, the highlights did not form CRs with the black matte walls. Moreover, the results failed to support any interaction between the highlights and the surrounding floor. The floor's relative achromatic color was correlated with its relative luminance in the room. [There was a slight, nonsignificant lightening of the black walls, as might be expected from the (very black) diffuse reflectance of the specular objects. The effect was too slight, however, to allow for generalizations.]

Other experiments, to be described shortly, will give a clearer picture of the role of specularity in achromatic constancy.

\section{A Stimulus Structure for}

\section{Absolute Achromatic Constancy}

In Figure 2, for the experiments on empty versus filled space, achromatic appearances of the black walls varied from very dark gray to light gray (Functions $A$ to $C$ ) or were bunched tightly around middle gray (Functions $\mathrm{G}$ to $\mathrm{J}$ ). This means there was no $\mathrm{ab}-$ solute achromatic constancy under any of these conditions. The black walls were seen to be gray, not black. Only $4 \%$ of the 528 achromatic responses in these experiments fell into the three black response categories.

Absolute achromatic constancy was little improved by other experimental arrangements. Under high diffuse illumination (Function L) and with the point source over the far wall (Function D), only 4 of the 96 responses were black responses.

In each of these failures of absolute achromatic constancy, the underlying physical events were illumination gradients, not reflectance differences. The underlying physical events were easily transformed, however.

The experiment with the black matte objects was rerun, with the black matte objects replaced by white ones. This change did not affect the luminances of the four walls. What the white matte objects did was to greatly expand the maximal range of median luminance between surfaces, which became 80 to 1 for the point source and 36 to 1 for the diffuse source. This contrasted to ratios of 2.2 to 1 and 1.2 to 1 , respectively, when black matte objects were in the room. Since the black walls were the denominators in the ratios of 80 to 1 and 36 to 1 , the black walls should appear black. Thus, absolute achromatic constancy was expected to occur.

These expectations were satisfied. The results are shown in Functions $F$ and $\mathrm{K}$. Both of the functions lie in the "black region" of Figure 2. For the first time, the black walls, on average, appeared black under both types of illumination. For the 22 subjects, $72 \%$ of their responses fell into the three black-response categories; the figure of $\mathbf{4 \%}$ was cited earlier for when the space was empty or contained black objects. Walls that had appeared gray (Functions $A$ to $C$ and $G$ to $J$ ) now appeared black, even though their surface luminances had not changed.

Relative achromatic constancy also occurred. This is shown by the flatness of Function $K$ in Figure 2 for diffuse illumination. Of the 22 subjects, 18 gave flat functions. Two of the remaining four subjects saw floor and far wall as the same. The other two subjects saw the floor, which had the highest wall luminance, as darker than the far wall, which had the second highest wall luminance. These two subjects provide the only support for interactive, coplanar contrast effects between the white objects and the black floor. Despite the relative coplanarity of 
the actual surfaces, there were no specific interactive effects.

Relative achromatic constancy did not occur under point-source illumination. The positive rise in Function $\mathrm{F}$ (of the 22 subjects, 17 gave rising functions) was very reliable [Friedman $\chi^{2}(3)=21, \mathrm{p}<.001$ ]. This is also a demonstration of the interrelatedness of absolute and relative achromatic constancy.

\section{.Three Current Experiments about Specular Effects}

It seemed appropriate to introduce here three recent experiments that will substantially complement this discussion. The three experiments are not listed in Table 2, but they were run under conditions identical to those of Experiments 1-4, viz, binocular viewing of the black matte room, point and extended sources, but judgments of three rather than four walls. Judgment of the right wall was omitted because in Experiments 1-8 its inclusion tended to give redundant information. The three experiments were distinguished by the fact that in each a different set of objects (white specular, middle gray specular, middle gray matte) was placed in the room. Three groups of 18 subjects participated.

In the first of the three experiments, the results with the white matte objects, described just above, were reproduced, using white specular objects (Munsell 9.2). Functions like $F$ and $K$ in Figure 2 resulted, although they were located slightly deeper into the black region. For the point and extended sources, $88 \%$ of all responses were black responses; they were $72 \%$ for the white matte objects. That was a further example of absolute achromatic constancy. (As in the case of the white matte objects, there was insufficient evidence to support coplanar effects between the white specular objects and the floor.)

In the two other recent experiments, matte or specular middle-gray objects were placed on the floor of the black matte room. Achromatic functions for the wall surfaces, when plotted in Figure 2, fell below Functions $A$ to $C$ and $G$ to $J$ and above Functions $F$ and $K$. This means that each black wall systematically shifted in apparent achromatic color from middle gray to dark gray to black as objects were varied from black to middle gray to white. (To demonstrate these effects, the Kruskal-Wallis test statistic $H$ was calculated, one each for the effect on each wall of the three classes of matte and the three classes of specular objects, under point and extended sources. These 12 values of $\mathrm{H}$ formed a frequency distribution, with a median of 34 , a range of 18 to 42 , and individual ps at least as small as .001 , with $2 \mathrm{df}$.) These findings also demonstrate the dependence of apparent achromatic color on CRs, for three-dimensional spaces. (As in the other experiments, no coplanar effects were found.)
The functions for the wall surfaces, produced by the middle-gray matte and specular objects, clarified to some extent the question: How do specular objects interact with the surrounding surfaces? Under diffuse illumination, when middle-gray matte and specular objects were in the room, the median judgments of the walls were virtually identical. This implies that it is the diffuse reflectance of a specular object that interacts with surrounding surfaces. This conclusion was supported as well, although to a lesser extent, by illumination with a point source. (This issue will be investigated more systematically in a separate paper.)

\section{Achromatic Constancy Within Specular Objects}

Absolute achromatic constancy tends to be inherent to specular objects. That was the claim made in the introduction. In order to test this hypothesis, four sets of objects (matte and specular, middle gray and white) were separately displayed on the floor of the black matte room. A group of 18 subjects was assigned to each set of objects (Experiment 9, Table 2). The room, binocularly observed, was illuminated by point and extended sources, as in Experiments 1-4. The subjects judged just the achromatic color of the objects.

The results are shown in Figure 3. Consider first the top two lines for matte objects. The upper line was for the point source, the lower one, for extended sources. The two data points anchoring the left of the two lines were for the middle-gray matte objects. Despite the actual color of middle gray, the objects appeared almost as white as the white matte objects, which were represented by the two data points anchoring the right of the two lines. [The rise of the upper, but not the lower, line gave a statistically significant Mann-Whitney $U(p<.05)$. In addition, the separation of the two points on the right, a within effect, was reliable, with a binomial split of $13-0$, 5 ties; the corresponding result for the two points on the left was 8-4, 6 ties.] In comparison, consider the second pair of lines for the specular objects. (The upper line was for the point source, as before.) They were relatively steep, whereas the lines for the matte objects were relatively flat. That difference indicated a degree of absolute achromatic constancy for the specular objects and none for the matte objects. [The separation of the two specular lines and their rise from middle gray to white were significant changes (ps $<.001)$.]

On the left, the two data points for the middlegray specular objects, under point and extended sources, are superimposed. Achromatic color did not alter, therefore, with a change in illumination gradients. That fact is another sign of achromatic constancy. These two data points, in the region of light gray, fell significantly below corresponding points for the middle-gray matte objects (Mann-Whitney Us gave $\mathrm{ps}<.001$ ). 


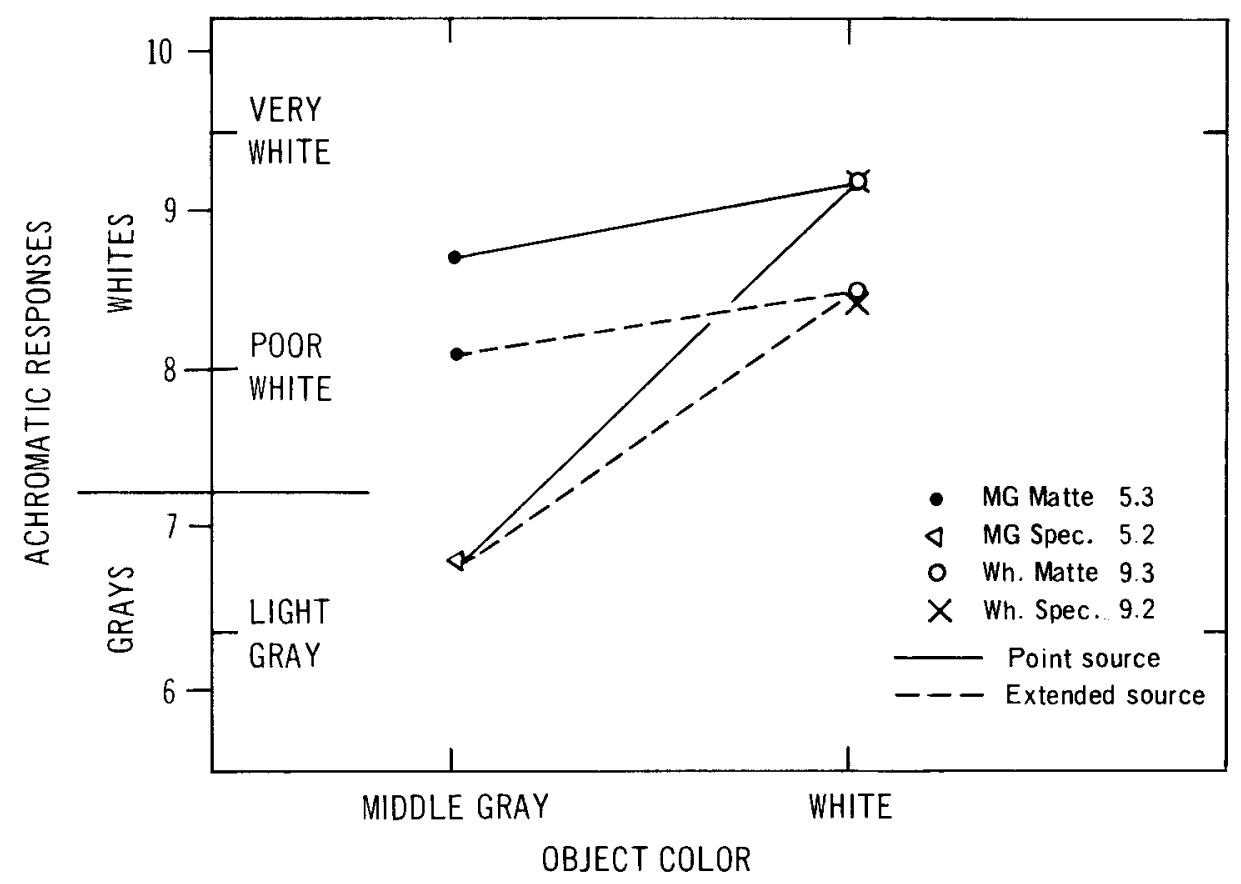

Figure 3. Achromatic responses to matte and specular objects, painted middle gray (MG) or white (Wh.).

Variability around the four median data points in Figure 3 for white objects was small: $94 \%$ and $100 \%$ of the responses were, respectively, in two and three adjacent response categories. For middle-gray objects, the corresponding points were $61 \%$ and $83 \%$. Another indicator of the 72 subjects' variability in Figure 3 was the proportion of white responses for the four object types: .25 for middle-gray specular, .78 for middle-gray matte, and $\mathbf{1 . 0}$ for both white matte and white specular.

In this paper, specularity must necessarily be confined to a limited role. The experiments were included to give some idea of the role that specularity can play in achromatic perceptions (see also, Flock, 1984).

\section{The Definition of Absolute}

\section{Achromatic Constancy}

Some data in Figure 2 were troublesome as regards a definition of absolute achromatic constancy. The claim has been made that absolute achromatic constancy tends not to occur if differences in surface luminance are caused only by illumination gradients. In Function $E$ in Figure 2, two of the black wall surfaces appeared black, even though only illumination gradients were present. These "black" responses may be conceptualized as instances of absolute lightness (achromatic) constancy. To consider them in that way, however, ignores the context in which they occurred. Function E describes a distribution of apparent achromatic color, from black to white, that has no relation to the actual reflectances of the surfaces.
We prefer a definition of absolute achromatic constancy that emphasizes an entire distribution of achromatic color. If a distribution of apparent achromatic color produces a reasonable fit to the actual distribution of reflectances, then, according to our definition, absolute achromatic constancy has prevailed. If there is a hopeless mismatch, as in the case of Function E, then absolute achromatic constancy has definitely not prevailed.

For the experiments in Figure 2 that involved illumination gradients, it should be clear that there was no absolute achromatic constancy, according to our definition; and there was very little even according to a definition that focuses on the response to single surfaces. (Function $\mathbf{M}$ for the white matte room, Experiment 11 , will be discussed later in this paper.)

\section{Response Variability for Experiments 1-8}

Response variability around each of the median data points in Figure 2 was relatively homogeneous. With the exception of Functions $\mathbf{F}$ and $\mathbf{K}$ (for white matte objects), total response variability was confined to approximately one and one-half response categories above and below each median value in the figure. That means that virtually all of the variability was confined to three adjacent response categories, such as very dark gray, dark gray, and middle gray. Excluding Functions F and K, 5\% of the total of 776 responses fell outside of three adjacent categories and $15 \%$ fell outside of two adjacent categories. Roughly, this makes a normal standard deviation 
equal to three-fourths of a response category. Thus, three-fourths of a response category to either side of any point in Figure 2 will locate about $68 \%$ of the responses. (The variant responses that fell outside of two and three response categories tended to be found equally under all experimental conditions.)

Variability was considerably greater for Functions $F$ and $K$ (for the white matte objects). In those two experiments, $29 \%$ of the 188 responses fell outside of three adjacent "black" categories, varying up through the grays to middle gray. This variability tended to be distributed uniformly over the four surfaces and the two illumination conditions; and virtually all of it was contributed by fewer than one-third of the subjects.

\section{The Reliability of the Functions in Figure 2}

The statistic, $\chi^{2} / \chi^{2} \max$, in the table of Figure 2 is a measure of the reliability of the upward rise of the functions. The ratio is the Friedman chi-square for a function over the maximum possible Friedman chisquare for that function. This ratio indexes the agreement among subjects in an experiment. It may vary from one to zero. When it is one, the subjects have perfectly agreed that the four surfaces are all achromatically different and that they differ in the same direction. When the ratio is zero, the subjects have perfectly agreed that all of the surfaces are exactly the same color (or, the subjects have perfectly disagreed as to the colors of the surfaces, an alternative not found in our data).

The ratios and the number $(\mathrm{N})$ of subjects for each experimental condition are given in the table in Figure 2. Notice that the ratios are relatively large for point-source illumination but relatively small (except for Function L) for extended sources. The probability associated with each of these ratios is the same as for its chi-square, with $\mathrm{df}=3$. (The chi-square is the product of the ratio and $3 \mathrm{~N}$. Thus, the first ratio, .80 , multiplied by $3(17)$ gives a $\chi^{2}$ of 41 , which, with $\mathrm{df}=3$, has a probability far less than .001 .)

For the proportions in the Figure 2 table, the probabilities associated with Functions $A$ to $F$ and $L$ are at least less than .001 . The probabilities associated with the remaining proportions for diffuse illumination are all greater than .05 . This means that there were no overall significant effects of luminance differences for diffuse illumination.

\section{Achromatic Effects of}

\section{Small Luminance Differences}

Despite the conclusion about overall effects of diffuse illumination in the last section, the values for chi-square for diffuse illumination mask a small, but sturdy, effect in the data. In explanation, it is necessary to consider the surface luminances under diffuse illumination.

Under diffuse illumination, several wall surfaces might have the same, or nearly the same, median lu- minance. The two side walls for Functions $G$ to $J$ had the same luminances; the floor and far wall had nearly the same luminances; but the side walls had lower luminances than the floor and far wall. As a result, the two side walls might appear the same; the floor and far wall might appear the same; but the side walls might appear reliably darker than the floor and far wall. That pattern of response would shift $\chi^{2} / \chi^{2}$ max toward statistical nonsignificance even though there might be a reliable effect within some of the comparisons. The results confirmed those expectations.

For 75 subjects who were involved in Functions $G$ to $J, 47$ reported all surfaces to be the same. (In view of the overall similarity of luminances among all of the surfaces, that is not an unexpected result.) Of the remaining 28 subjects, 26 reported the side walls to be the same, 18 reported the floor and far wall to be the same, and all reported the side walls to be darker than the other two walls. This was a 28-0 split, with no reversals. One must conclude from this that, under these diffuse illumination conditions, there is a systematic effect of luminance on achromatic appearances, at least for 28 of the subjects or $37 \%$ of the group.

That result disappeared in Function $\mathbf{K}$ for the white matte objects, even though surface luminances had not changed. It is as if a marginally detectible luminance difference had effects when the white objects were absent, but was ignored when the white objects were present. If that is true, it could have some theoretical importance. It could be interpreted to mean that small luminance differences are minimized or ignored, given the presence of other larger differences, but have increasing achromatic effects as other larger luminance differences are scaled down in magnitude.

Under point-source illumination, some walls may also have the same or nearly the same luminance, as in fact was the case for some of the conditions. Responses should then be the same and the magnitude of the ratio, $\chi^{2} / \chi^{2}$ max, should decrease. Except for that fact, the ratios in Figure 2, which are already reasonably large for the point-source conditions, might have been much larger.

\section{Centers of Achromatic Distributions}

Achromatic color perceptions did not depend on absolute luminance levels. In explanation, draw a horizontal line at the level of middle gray $(Y=5.0)$ in Figure 2. The line intersects all functions except $F$, $K, M$, and $N$. From one intersection to the next, reading left to right, luminance increases, but the achromatic appearance of middle gray remains unchanged. From experiment to experiment, therefore, achromatic appearances did not vary with luminance.

There is a second feature to the points of intersection. Each tends to lie somewhere toward the middle of its achromatic distribution. It is as if the achro- 
matic responses were centered on middle gray. As a rough demonstration of this, the median achromatic responses at the end points of each line graph were arithmetically averaged. The result was a set of centers: A, 4.4; B, 4.4; C, 4.4; D, 4.4; E, 4.7; G, 5.0; $\mathrm{H}, 5.0 ; \mathrm{I}, 5.0 ; \mathrm{J}, 5.0 ; \mathrm{L}, 5.1$. All of these averages were clustered at or near middle gray.

Center values were also determined for Functions $\mathrm{F}$ and $\mathrm{K}$ by averaging the function's lowest response with the response to the white matte objects from Experiment 9 . The two center values were 5.48 (point source) and 5.42 (diffuse sources).

One other center was desired, for Function $\mathrm{N}$ in Figure 2. Function $\mathbf{N}$ (to be described shortly) locates median responses under reduction to the black and white far walls, from five different experiments (Experiments 5-8 and 11, Table 2). One of the data points $(\triangleleft$ in Function N, Figure 2) was selected to be replicated (Experiment 10, Table 2). At the same time, the 10 new subjects were asked to judge the blackness of the dark surround. The two median responses were 7.88 , or poor white, for the far wall and 1.86 , or black, for the dark surround. These averaged to 4.9.

Excluding Function $\mathbf{M}$, the functions in Figure 2 were centered at or near middle gray, as Helsonian theory would have predicted. This result might seem trivial. What else, one might ask, would the average of a phenomenal black and white be but middle gray? Notice, however, that the same centering tendencies occurred in Functions $G$ to $J$, which were remote from black and white.

\section{CRs as a Stimulus for Achromatic Differences}

Aside from the centering pattern in the data, there was a second striking characteristic about the line graphs in Figure 2. The lines tended to be parallel at each level of the graph. In explanation, draw horizontal lines at dark gray, middle gray, and light gray, in Figure 2. Within each "zone," the lines are relatively parallel. This means that within achromatic zones the amount of achromatic change tended to be proportional to the rate of change in luminance. (Function $\mathrm{K}$ was a clear exception, being flat. As explained earlier, when there were large luminance differences, like those produced by white objects and black walls, then small luminance differences tended to be ignored or overlooked, or to have decreasing achromatic effects.)

Figure 4 clarified these proportional relations between achromatic responses and luminances. The figure was produced by using the Helsonian methods described in detail in the introduction. The median surface luminances for each illumination condition were arithmetically averaged to give a representative overall average luminance for the scene (ROLS). Each surface luminance in the scene was next expressed as a ratio of the ROLS, to give the CR for that surface. Plotting achromatic appearance of a surface against its CR (expressed in logs) gave Figure 4. (All of the data from Figure 2, except for Functions $M$ and $N$, were utilized to produce Figure 4.) The closeness of the relation between achromatic appearances and lu minance ratios is described by the line drawn through the 52 median data points and by a Spearman correlation coefficient, which was $\mathbf{9 5}$.

Figure 4 integrated results that derived from three different classes of visual stimuli. These stimuli were produced in turn by three uniquely different physical conditions: illumination gradients and reflectance differences between matte surfaces and within specular surfaces. As a first approximation, CR in Figure 4 seems to give a persuasive explanation for differences in achromatic responses. The close relationship in Figure 4 supports the claim that CR is at least a major determining stimulus for achromatic perceptions. It is the CR itself that is the stimulus, not the very different physical conditions that give rise to it or the different visual structures in which it is embedded. This claim for CRs is widely held, although it has not always been well documented by empirical data for three-dimensional scenes.

Three theories, dependent on the concept of CR, were described in the introduction. Helsonian theory was directly applied to the data, thereby explaining the patterning of data in Figure 2 and producing the systematic relation found in Figure 4 . A result equally as good as that in Figure 4 occurred when CR for each surface was formed by using its surrounding background luminance (Wallach, 1948) instead of an average for the overall scene (Helson, 1964). Nevertheless, if there has been a preference for Helsonian theory in this paper, it is because of Helson's expectation that achromatic distributions, in general, will tend to be centered on middle gray. That result was a compelling feature of our data, as shown in Figure 2.

It was not possible to generate specific theoretical predictions, using Land and McCann's (1971) concept of edge ratios. The luminance patterns on the walls and between the walls of the black matte room were too complicated for that. Even so, we had reservations about their edge ratios. The idea seemed to require too much from a visual system. It seemed to require uniform sensitivity for, and almost simultaneous registration, integration, and processing of, every adjacent luminance variation, everywhere on the retina, for an entire scene, however complex. In the absence of these simultaneities, the visual system would have to process sequentially, continuously changing and adjusting both the values of CRs and their corresponding achromatic effects. That would imply that apparent achromatic colors in a scene were unstable and continuously labile. That implication, we believe, has little or no truth value. When one enters a scene, achromatic colors appear instantly stable, no matter how complex the scene and how slow the visual scan. Moreover, as Land and McCann 


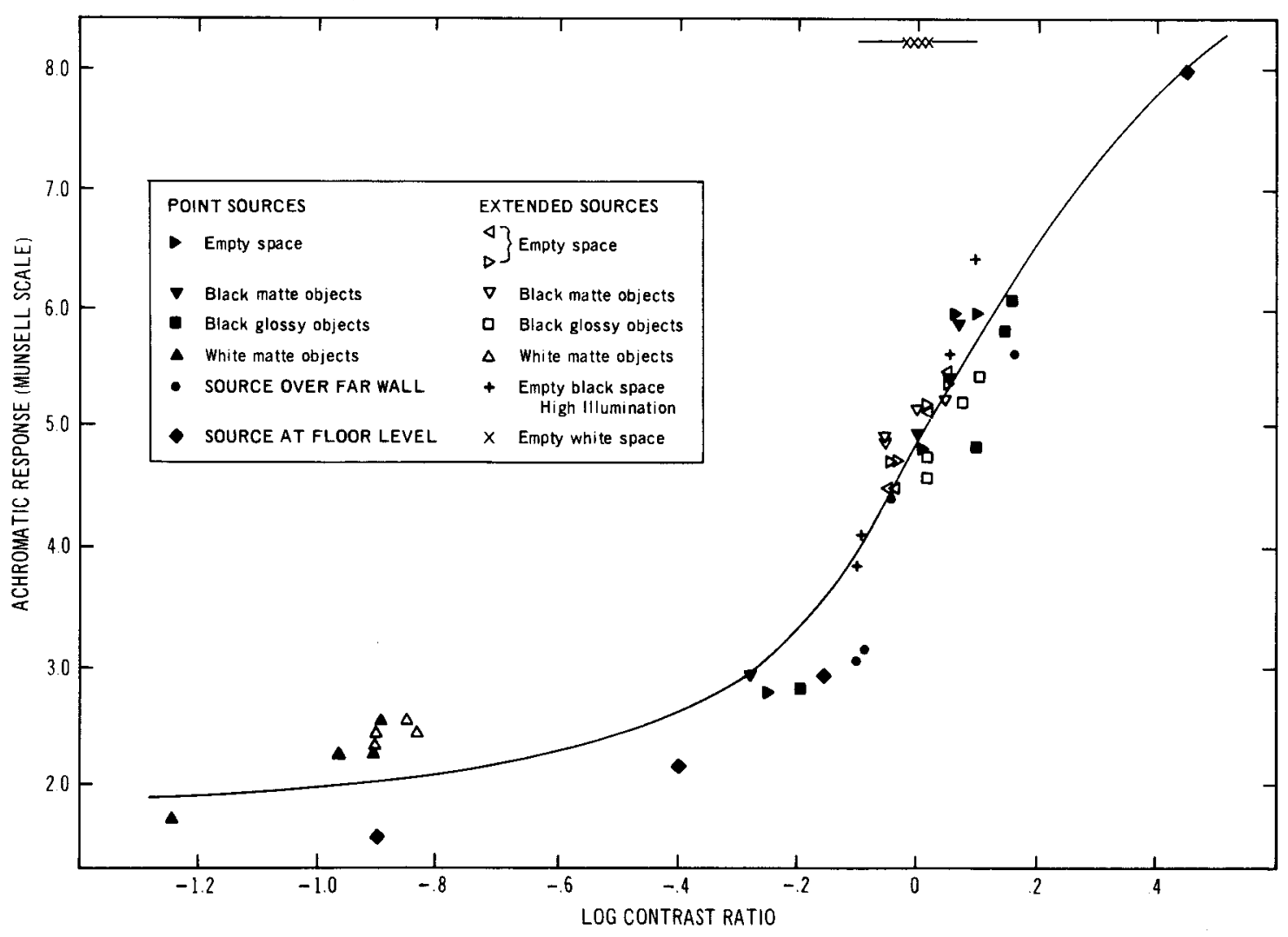

Figure 4. Fifty-two median achromatic responses to four surfaces from 13 different experimental conditions plotted against the contrast ratio (CR) in logs for each surface.

stated their theory, it is not clear how the theory would negotiate specular surfaces, dihedral intersections, shadows, etc. Even so, what is essentially important is the focus on CRs (ratios of luminance) as the explanatory concept for achromatic perception.

\section{Apparent White}

In several of the experiments described above, phenomenal white occurred as the result of CRs. When CRs had sufficient magnitude, the surface in the numerator of the largest ratio appeared white. This occurred when the middle gray and white objects were placed in the black matte room. Although phenomenal white depended on CRs in those experiments, in two other experimental instances of apparent white, CRs could not account for the results. In binocular observation of the empty white matte room (Function $M$ in Figure 2) and reduction of the black and white far walls (Function N, Figure 2) CRs were trivial in magnitude or could not be specified. The CR structure was "null."

In observing the white matte room, each of the 14 subjects reported that the four white walls appeared the same white ( 3 said white; 11 said poor white). Finding that the empty white matte room appeared white presented a puzzle. Why did it not appear mid- dle gray, like the diffusely illuminated black matte room? With median luminances very similar from one wall to another, the apparent achromatic color of the white walls should have clustered at middle gray. That, of course, did not happen. An alternative explanation would make use of the darkness of the inside of the viewing hood. With that darkness in the denominator and the white matte room in the numerator of a CR, a seemingly appropriate $C R$ could be fabricated. The white room appeared just as white, however, when the viewing hood was removed and the room was observed from the brightly illuminated outer space. The point is, there was no specifiable surround luminance, and thus, perhaps, no CR.

Consider next the experiments with reduction screens (Function N, Figure 2). The far wall was physically black for 52 subjects and physically white for 14 subjects. The wall was illuminated as it was for Functions $G, D, L$, and $M$ in Figure 2. Under reduction, the far wall became a 10-deg disk of homogeneous light, centered in a totally dark reduction screen. The luminance of the five reduction disks in Function $N$, Figure 2, varied over one log unit, or by a factor of 10 , from $-0.77 \log \mathrm{fL}$ to $0.23 \log \mathrm{fL}$. Over this 10 times change in the light, there was a small variation in the subjects' responses. Of the 66 subjects, 49 said poor white, 14 said white, and 3 said very light gray. 
The slight rise in Function $\mathrm{N}$ in Figure 2 occurred because the "white" responses occurred to the higher luminances and the three "very light gray" responses occurred to the lower luminances.

The reduction conditions might seem to fit the paradigm for CRs. There was an inlying disk of light with a luminance that varied from .17 to $1.7 \mathrm{fL}$ for the different illumination conditions. There was the surrounding screen that had a very low luminance and appeared black. One might conclude that the inlying disk appeared white because of the CR between the disks and the surrounds.

Actually, the concept of CR cannot easily be applied to the reduction experiments. For one thing, the luminance of the surrounding screen could not be specified. The photometer, with a 6-min probe, could not record below $.001 \mathrm{fL}$. The screen's luminance, had it been measurable, would have been well below that value. Second, even assuming an arbitrary value of $.001 \mathrm{fL}$ for the screen, the CRs for the inlying disk would have been extremely large in magnitude, varying from 170 to 1 up to 1,700 to 1 . Despite those large CRs, the disks appeared on average only poor white. One might have expected the disks to appear much whiter and, as CR was varied from 170 to 1,700 , to have appeared super white. That did not happen. Third, the reduction of the black and white walls and the binocular observation of the white room provided very different and very disparate physical conditions. Despite that, as indicated by Functions $M$ and $N$ in Figure 2, the achromatic responses seemed to be following the absolute luminances of the targets. That was a tempting conclusion, which we have resisted. The achromatic appearance of white for those various disparate conditions did depend, however, on something other than the relative luminances that had prevailed in the other experiments in this paper. That something may have been the homogeneity of their luminances.

In explanation, the white surfaces in the white room functioned as diffuse sources for other white surfaces, reflecting a great amount of light back and forth. The multiple reflections wiped out any luminance differences that might have been present because of surface texture and illumination gradients. The white room, for example, was illuminated by a $5-W$ point source on the back side of the viewing hood. Despite that, it was as if the white room had been everywhere diffusely illuminated. In contrast to this, consider the black walls. In the absence of homogenizing effects from multiple reflections (black walls under moderate illumination reflect too little light), the surface texture and illumination gradients on the black surfaces yielded heterogeneous luminances.

Under reduction conditions, however, the luminances of the black far wall appeared homogeneous. This was established by having the 66 subjects judge the distance, which was $196 \mathrm{~cm}$ to the target disk.
The subjects' median and modal judgment was $30 \mathrm{~cm}$, which was the approximate distance to the edge of the farther reduction aperture. The eye, therefore, was focused on the edge of the aperture, not on the target. With the target out of focus, its light was homogenized. (The same 66 subjects under binocular observation judged the distance of $168 \mathrm{~cm}$ to the far wall to be a median distance of $120 \mathrm{~cm}$.)

Part of the reason, then, why the reduction disks and the surfaces in the white matte room appeared white was the homogeneity of their luminances. To complete the explanation, this discussion of apparent white has to be placed in the context of the general theory described in this paper. To do that, three empirically testable assumptions that have been more or less explicit throughout this paper are required. The assumptions include concepts of achromatic segregation and of the centering of achromatic distributions.

The first assumption asserts that the visual system selectively segregates those surfaces that are seen to be illuminated by the same sources or to derive or generate their light in the same way. Provided the surfaces have some spatial coherence, it doesn't matter how they are arranged spatially. A set of such surfaces will be called an achromatically segregated entity. Examples of such entities might be the surfaces in a sunlit scene, in one's living room, or in the white and black matte rooms, empty or filled, that were used in the present research. Single surfaces such as a reduction disk or, separately, its surrounding darkness are other examples. Within a spatial arrangement, part of the space might be under a large cast shadow, as, for example, that caused by a bank of clouds or a glade of trees on a sunlit day. These shadowed subsets would be registered, presumably, as being under illumination that was different from that of the remainder of the scene. Self-luminous primary sources are also presumed to be separate subsets. The diffuse reflectance of specular objects can, it seems, concurrently belong to more than one achromatic entity. In explanation, in one of the above experiments, the diffuse reflectance of a middle-gray specular object formed CRs with the black matte walls of the room, darkening the walls (see page 122). The same diffuse reflectance also interacted with its own specular highlights (i.e., its mirror images) to produce a degree of absolute achromatic constancy of the object (Experiment 9).

This first assumption is implicit in most perceptual research that uses viewing hoods, apertures, and other arbitrary devices to separate a display from some larger scene. Implicit in such arrangements is the experimenter's expectation that the visual system will naturally, without instruction or strain, accept the delineated scene as separate and segregated from the larger laboratory scene. Interactions other than momentary transients are not expected, are presumed 
not to occur, and probably do not occur between the segregated regions.

A famous example of the experimental manipulation of an achromatically segregated entity is that of Gelb (1929). He suspended a black disk, specially illuminated, in a dimly lit room. Disk and room were seen to be illuminated the same, even though they were not, and a CR was formed between them. That CR was broken by placing a small bit of white paper on the face of the disk. The disk and bit of paper then formed a CR and were seen to be illuminated differently from the rest of the room.

The first assumption, then, establishes the concept of achromatically segregated entities. What is common within each of these entities is a visual registration that the included surfaces are illuminated by common sources or derive or generate their light in the same way. The second assumption concerns CRs. They are primarily formed only within an achromatically segregated entity. This assumption follows from the first. It explains, for example, why specular highlights did not interact with surrounding walls, but did influence the apparent color of the specular object.

The third assumption already has been made explicit. Individual matte surfaces that are heterogeneous in luminance, that belong to an achromatically segregated entity, and that have similar median luminances will give achromatic distributions centered at and clustered around middle gray (Functions $\mathbf{G}$ to $\mathrm{J}$, Figure 2). If the luminances over the individual surfaces are made more and more homogeneous, the achromatic distribution will be centered on a lighter and lighter achromatic color. When the luminances are made very homogeneous, the achromatic distribution will be centered on white (Functions $M$ and N, Figure 2). At any one of these levels, introducing CRs will spread the achromatic distribution toward white in one direction and toward black in the other direction (Functions A to E, Figure 2). If the center point is white, the distribution, presumably, would asymptote on super white (or become luminous) in one direction and shift toward grays and blacks in the other direction. Increasing the magnitude of CRs will, however, tend to shift the center point of an achromatic distribution toward middle gray.

If this third assumption is correct, it has implications that are easily tested. A room composed of black matte surfaces might be diffusely illuminated. Then as the diffuse illumination was made more intense (Function L, Figure 2) and made to emanate from an increasingly larger area, the center of the achromatic distribution should systematically shift upward toward white. A phenomenally white surface that is not dependent on a CR for its whiteness is simply an end point of a process of homogenization of luminances. Surface texture and illumination gradients are both involved in generating heterogeneous luminances. Reducing surface texture should have an influence, therefore, on the center point of an achromatic distribution. (In this discussion, the third assumption has necessarily been confined to matte surfaces. In the absence of research using specular rooms, it is not possible at present to generalize beyond matte surfaces.)

\section{GENERAL DISCUSSION}

Different visual structures that might affect achromatic perceptions were hypothesized. The structures were identified by reference to their determining conditions: primary illuminating sources and the reflecting properties of matte and specular surfaces. For an illuminated three-dimensional space, two features were common to the different visual structures. First, there was a measurable representative luminance for a surface and for surfaces collectively. Second, there was a measurable contrast-ratio (CR) for each surface. A surface's CR was formed by taking the ratio of its median luminance to the representative overall luminance for the scene (ROLS). A number of simple relations between these CRs and achromatic responses were demonstrated by the experiments in this paper.

One relation, common to all of the experiments, was the result that a CR, however it was produced, had lawful achromatic effects. The CR may have been produced solely by the effects of the illumination or by a difference in reflectance among matte surfaces, and within the area of a specular surface. Regardless of the cause, the resulting CRs were correlated to differences in achromatic appearances. Moreover, the CRs and their interactive effects were independent of any particular spatial arrangement. Strong, even dramatic, interactive contrast effects occurred among surfaces, whatever their spatial arrangement. Black walls that appeared middle gray, for example, shifted in appearance to black, simply by placement of a few white objects on the floor of the room. These interactive contrast effects did not depend on specific spatial concepts like the coplanarity of surfaces, degrees of adjacency of surfaces, or disk-annulus relations. Such spatial ideas were irrelevant within the three-dimensional arrangements of these experiments.

A second important result indicated that distributions of achromatic responses tended to be centered at or near middle gray (for surfaces with heterogeneous luminances). Again, it did not matter how the CRs, from which an achromatic distribution derived, were produced. When the magnitude of the CRs was small (i.e., diffuse illumination of surfaces with the same reflectance), all of the responses tended to cluster near middle gray. When the magnitude of the CRs was larger (i.e., point-source illumination of surfaces with the same reflectance, surfaces with different reflectances, or specular surfaces), then the 
achromatic distributions spread away from middle gray toward white and black.

A third result pertained to the accuracy of achromatic perceptions. Perceptions were in excellent correspondence with the CRs as proximal stimuli. Perceptions were not necessarily in good correspondence with the reflectances of distal surfaces, unless those surfaces were specular. When, for example, black matte surfaces with the same reflectance were illuminated by one or the other point source, neither absolute nor relative achromatic constancy occurred. No mediating process intervened to interpret the CRs and create achromatic (lightness) constancy. Whether a perception was "correct" with regard to distal surfaces depended on the kind of physical event that produced the CRs. The nature of the physical event, not its complexity, determined whether achromatic constancy would occur. In both a matte and specular world, achromatic constancy was hypothesized to be a probabilistic event, contingent on the probability that the scene contained specular objects or matte objects with a substantial range of reflectances. When the experimental scene contained those objects, absolute achromatic constancy did in fact tend to occur. The belief that a great deal of structure and complexity in the visual scene will in themselves suffice to create achromatic constancy was found to be untrue.

A fourth result pertained to phenomenal white, which could result from two very different processes. In one outcome, surfaces appeared white when the median luminance of the surface was in the numerator of a relatively large CR. Surfaces also appeared white if each surface was very homogeneous in luminance and the surfaces had similar median luminances. Under those conditions, the achromatic distribution was centered on white, not on middle gray as in the case of surfaces with heterogeneous luminances.

The experimental results in this paper defined a general theory of achromatic perception that is dependent on the concept of CR and on three assumptions. The CRs that produced Figure 4 depended on arbitrary spatial-temporal restrictions. The luminances forming the CRs were designated by the arbitrary boundaries of the experimental chamber. Light that might have been spatially or temporally outside of those boundaries was ignored. Moreover, if the correspondences in Figure $\mathbf{4}$ are considered as evidence, then the spatial-temporal restrictions that were imposed by the experimental arrangements were apparently appropriate. This logic suggests that visual systems form CRs and experimenters conceptualize CRs within the confines of coherent achromatic entities, segregated from other such entities. An entity is formed, we believe, because the surfaces within it are registered as being illuminated by com- mon sources or as generating and deriving their light in the same way.

In our experiments there were a number of such entities: the black matte room, the same room filled with black, white, or middle-gray matte objects, the empty white matte room, the disks of light segregated from the surrounding darkness (reduction conditions), and probably the highlights seen on specular objects. The first and second assumptions, therefore, were that the visual system processes CRs and achromatic colors within achromatically segregated entities. Within such an entity, all surfaces are registered as being illuminated by common sources or as deriving and generating their light in the same way.

The third assumption had to do with the centers of distributions of apparent achromatic colors. When luminances on individual surfaces were heterogeneous, because of surface texture or illumination gradients, the distribution of apparent achromatic color was centered on middle gray. As the luminances of the surfaces were made very homogeneous, the center point shifted upward to white. Presumably, as luminances are shifted from very heterogeneous to very homogeneous, center points of achromatic distributions will shift from middle gray to white. (The three assumptions, summarized here, were supported by the experiments in this paper.)

The theory of achromatic perception set forth in this paper and the experiments that have defined the theory have been based on what perceivers "see," not necessarily on what they "know." In the experimental procedures, subjects were encouraged to believe that surfaces might actually be the same color, different colors, or some the same and some different. This procedure prevented the subjects from taking the attitude that the experimental walls must be the same color because walls in a room are generally the same color. With that conventional schema, perceivers might ignore achromatic differences or even not "look" at surfaces. Our procedures forced subjects to look at each wall surface and to set aside any preconceived internal representation of the space. This cognitive uncertainty would be an absolute requirement in any replication of a data configuration like that of Figure 2. (On the subject of replication, Figure 4 would require a good set of light measures. That means a carefully worked out set of instrumental procedures and a sophisticated measuring instrument.)

\section{REFERENCES}

Beck, J. (1964). The effect of gloss on perceived lightness. American Journal of Psychology, 58, 54-63.

BECK, J. (1965). Apparent spatial position and the perception of lightness. Journal of Experimental Psychology, 69, 170-179.

BECK, J. (1981). Highlights and the perception of glossiness. Perception \& Psychophysics, 30, 407-410.

Flock, H. R. (1972). Toward a theory of brightness contrast. 
In M. H. Appley (Ed.), Adaptation level theory. New York: Academic Press.

Flock, H. R. (1974). Stimulus structure in lightness and brightness experiments. In R. B. MacLeod \& H. L. Pick (Eds.), Perception: Essays in honor of James J. Gibson. Ithaca and London: Cornell University Press.

Flock, H. R. (1982). Empirics versus beliefs in the visual world of achromatic contrast. Paper presented at the annual meeting of the American Psychological Association, Washington, D.C.

Flock, H. R. (1983, November). Unique visual structures for relative and absolute lightness constancy. Paper presented at the annual meeting of the Psychonomic Society, San Diego.

Flock, H. R. (1984). Illumination: Inferred or observed? Perception \& Psychophysics, 35, 293.

Gelb, A. (1929). Die "Farbenkonstanz" der Sehdinge. In A. Bethe et al. (Eds.), Handbuch der normalen und pathologischen Physiologies (Vol. 12, Part I). Berlin: Springer.

Gilchrist, A. (1977). Perceived lightness depends on perceived spatial arrangement. Science, 195, 185-187.

GILCHRIST, A. (1979). The perception of surface blacks and whites. Scientific American, 240, 112-124.

Gilchrist, A. (1980). When does perceived lightness depend on perceived spatial arrangement? Perception \& Psychophysics, 28, 527-538.

Gilchist, A., Delman, S., \& Jaconsen, A. (1983). The classification and integration of edges as critical to the perception of reflectance and illumination. Perception \& Psychophysics, $33,425-436$.

Gilchrist, A., \& JACOBSEN, A. (1984). Perception of lightness and illumination in a world of one reflectance. Perception, 13, 5-19.

Helson, H. (1938). Fundamental problems in color vision. I. The principle governing changes in hue, saturation, and lightness of non-selective samples in chromatic illumination. Journal of Experimental Psychology, 23, 439-476.

Helson, H. (1947). Adaptation-level as a frame of reference for prediction of psychophysical data. American Journal of Psychology, 60, 1-29.
Helson, H. (1964). Adaptation-level theory: An experimental and systematic approach to behavior. New York: Harper. HERING, E. (1964). Outlines of a theory of the light sense (L. M Hurvich \& D. Jameson, Trans.). Cambridge, Mass: Harvard University Press. (Original work published 1905, 1907, 1911)

Hess, C., \& PreTori, H. (1970). Quantitative investigation of the lawfulness of simultaneous brightness contrast (H. R. Flock \& J. H. Tenny, Trans.). Perceptual and Motor Skills, 31, 947. 969. (Original work published 1896)

Hunte R, R. S. (1975). The measurement of appearance. New York: Wiley.

KaRDOS, L. (1934). Ding und Schatten. Zeitschrift fuir Psychologie, No. 23.

KATZ, D. (1935). The world of colour (R. B. MacLeod \& C. W. Fox, Trans.). London: Kegan, Trench, Trubner. (Original work, 2nd ed., published 1930)

KoFfKA, K. (1935). Principles of gestalt psychology. New York: Harcourt Brace. American, 237, 108-128.

LAND, E. H., \& MCCANN, J. J. (1971). Lightness and retinex theory. Journal of the Optical Society of America, 61, 1-11.

Leibowitz, H., Mote, F. A., \& Thurlow, W. R. (1953). Simultaneous contrast as a function of the separation between test and inducing fields. Journal of Experimental Psychology, 46, 453-456.

Mershon, D. (1972). Relative contributions of depth and directional adjacency to simultaneous whiteness contrast. Vision Research, 12, 969-979.

WALLACH, H. (1948). Brightness constancy and the nature of achromatic color. Journal of Experimental Psychology, 38, 310-324.

Wallach, H. (1963). The perception of neutral colors. Scientific American, 208, 107-116.

(Manuscript received September 9, 1983; revision accepted for publication May 21, 1984.) 